\title{
A phase 2 study of adjuvant carboplatin plus S-1 followed by maintenance S-1 therapy for patients with completely resected stage II/IIIA non-small cell lung cancer-Japanese Northern East Area Thoracic Surgery Study Group JNETS1302 study
}

\author{
Takeo Hasegawa ${ }^{1,2}$, Hiroyuki Suzuki ${ }^{1,2}$, Jiro Abe ${ }^{1,3}$, Akira Sakurada ${ }^{1,4}$, Chiaki Endo ${ }^{1,4}$, Nobuyuki Sato ${ }^{1,5}$, \\ Tohru Hasumi ${ }^{1,6}$, Hiroyuki Deguchi ${ }^{1,7}$, Hiroyuki Oura ${ }^{1,8}$, Satomi Takahashi ${ }^{1,3}$, Hajime Saito ${ }^{1,7}$, \\ Hidetaka Uramoto ${ }^{1,9}$, Motoyasu Sagawa ${ }^{1,10}$, Yoshinori Okada ${ }^{1,4}$
}

${ }^{1}$ Japanese Northern East Area Thoracic Surgery Study Group, Seiryomachi, Sendai Aoba-ku, Japan; ${ }^{2}$ Department of Chest Surgery, Fukushima Medical University, Hikarigaoka, Fukushima, Japan; ${ }^{3}$ Division of Thoracic Surgery, Miyagi Prefectural Cancer Center, Medeshimashiote Nodayama, Natori, Japan; ${ }^{4}$ Department of Thoracic Surgery, Tohoku University, Seiryomachi, Sendai Aoba-ku, Japan; ${ }^{5}$ Division of Thoracic Surgery, Aomori Prefectural Central Hospital, Higashitsukurimichi, Aomori, Japan; ${ }^{6}$ Division of Thoracic Surgery, Sendai Medical Center, Miyagino, Sendai Miyagino-ku, Japan; ${ }^{7}$ Department of Thoracic Surgery, Iwate Medical University, Uchimaru, Morioka, Japan; ${ }^{8}$ Division of Thoracic Surgery, Iwate Prefectural Central Hospital, Ueda, Morioka, Japan; ${ }^{9}$ Department of Thoracic Surgery, Kanazawa Medical University, Daigaku, Kahokugun Uchinadamachi, Japan; ${ }^{10}$ Division of Endoscopy, Tohoku Medical and Pharmaceutical University, Komatsushima, Sendai Aoba-ku, Japan Contributions: (I) Conception and design: H Suzuki, M Sagawa, C Endo; (II) Administrative support: H Suzuki, Y Okada; (III) Provision of study materials or patients: All authors; (IV) Collection and assembly of data: T Hasegawa; (V) Data analysis and interpretation: All authors; (VI) Manuscript writing: All authors; (VII) Final approval of manuscript: All authors.

Correspondence to: Hiroyuki Suzuki. Department of Chest Surgery, Fukushima Medical University School of Medicine, 1 Hikarigaoka, Fukushima 960-1295, Japan. Email: hiro@fmu.ac.jp.

Background: The standard adjuvant chemotherapy regimen for completely resected pathological stage II/IIIA non-small cell lung cancer (NSCLC) is four courses of cisplatin plus vinorelbine. However, the continuity and toxicity of cisplatin-based regimens remain problematic. Conversely, carboplatin-based chemotherapy is a less toxic and more tolerable regimen for various stages of NSCLC. In particular, the efficacy and tolerability of carboplatin plus S-1 in advanced NSCLC were confirmed by previous pivotal studies such as the LETS trail. Therefore, this phase II study assessed the feasibility, safety, and usefulness of carboplatin plus S-1 followed by maintenance S-1 as an adjuvant treatment.

Methods: In this single-arm, multicenter phase II study, 40 patients who previously underwent complete resection of NSCLC were enrolled from November 2013 to January 2015. The chemotherapy protocol was four cycles of carboplatin (AUC 5 on day 1$)$ and oral S-1 $\left(80 \mathrm{mg} / \mathrm{m}^{2}\right.$ every other day from days 1 to 21) followed by oral S-1 $\left(80 \mathrm{mg} / \mathrm{m}^{2}\right.$ every other day for 48 weeks). The primary endpoint was the treatment completion rate, and the secondary endpoints were adverse events and 2-year recurrence-free survival.

Results: The treatment completion rate of the planned schedule was as low as 30.0\% (90\% confidence interval: 40.3-63.0\%). The reasons for adjuvant chemotherapy discontinuation were adverse events, refusal, tumor recurrence, and other reasons in 13, 6, 10, and 2 patients, respectively. The 2-year progressionfree survival rate was $66.7 \%$ among patients who completed maintenance chemotherapy. There were no treatment-related deaths, and most adverse events were less than grade 3 .

Conclusions: Carboplatin plus S-1 followed by S-1 maintenance for 1 year in the adjuvant treatment of NSCLC was not tolerable, although most adverse events were not severe. However, patients who can fully complete the regimen might experience clinical benefit.

Keywords: Non-small cell lung cancer (NSCLC); carboplatin; S-1; adjuvant chemotherapy 
Submitted Jan 31, 2020. Accepted for publication Jun 11, 2020.

doi: $10.21037 /$ jtd-20-715

View this article at: http://dx.doi.org/10.21037/jtd-20-715

\section{Introduction}

Lung cancer is the leading cause of cancer death worldwide, and the recognized evidence-based standard adjuvant chemotherapy regimen for completely resected pathological stage II/IIIA non-small cell lung cancer (NSCLC) is four courses of cisplatin-based doublet chemotherapy (1). However, adjuvant cisplatin plus vinorelbine only prolongs overall survival (OS) by approximately $5 \%$ (1), whereas the completion rate of adjuvant cisplatin-based chemotherapy is unacceptable. In fact, the completion rate of four courses of cisplatin plus vinorelbine was $50 \%$ in the ANITA trial of patients with stage IB, II, or IIIA NSCLC (2). Similarly, the completion rate of four courses of chemotherapy was $45 \%$ in the JBR 10 trial of patients with completely resected pathological stage IB or II NSCLC (3). Greater efficacy can be achieved if the treatment completion rate of adjuvant chemotherapy is improved. Conversely, adjuvant chemotherapy using carboplatin-based regimens is relatively easy to administer in clinical practice because carboplatin is less toxic than cisplatin (4). In the LETS trial, the OS of carboplatin plus S-1 was not inferior to that of carboplatin plus paclitaxel, which is one of the standard regimen for unresectable advanced NSCLC, and the relative dose intensities of carboplatin and $\mathrm{S}-1$ in this trial were as high as $83 \%$ and $94 \%$, respectively (5). In the carboplatin plus S-1 group in the LETS trial, carboplatin (AUC 5) was given intravenously on day 1 , and $\mathrm{S}-1\left(80 \mathrm{mg} / \mathrm{m}^{2}\right)$ was given orally on day $1-14$. The toxicity of carboplatin plus S-1 group was not severe than carboplatin and paclitaxel group especially about alopecia and peripheral neuropathy (5). Thus, this study suggested that the carboplatin and S-1 combination was well tolerated and suitable in the adjuvant setting. Furthermore, most clinicians believe there are no major differences between cisplatin- and carboplatin-based regimens in the adjuvant setting (6). Meanwhile, several studies found that additional maintenance therapy after platinum doublet chemotherapy contributes to improved outcomes for patients with advanced NSCLC (7-9). However, the efficacy of maintenance chemotherapy after conventional adjuvant chemotherapy for completely resected pathological stage II/IIIA NSCLC has not been confirmed. Regarding potentially effective maintenance adjuvant chemotherapy for patients with NSCLC, S-1 is a feasible treatment candidate because the drug can be administered orally.

S-1 (TS-1; Taiho Pharmaceutical) is an oral fluoropyrimidine agent consisting of tegafur, 5-chloro2,4-dihydroxypyridine (CDHP; gimeracil), and potassium oxonate in a molar ratio of 1:0.4:1 (10). Classically, 5 -fluorouracil $(5-\mathrm{FU})$ is not active against primary lung cancer because most lung cancers express high levels of dihydropyrimidine dehydrogenase (DPD). However, S-1 is effective against lung cancers because CDHP inhibits DPD activity; thus, S-1 is a DPD-inhibitory fluoropyrimidine. $5-\mathrm{FU}$ is activated via phosphorylation, and the active metabolites kill tumor cells mainly through the inhibition of thymidylate synthase, a key enzyme in de novo DNA synthesis (11). S-1 was designed to exert a stronger antitumor effect than tegafur-uracil. A phase II trial of S-1 monotherapy as a first-line treatment for patients with advanced NSCLC reported a $22 \%$ response rate, and S- 1 adjuvant chemotherapy after surgery improved OS in patients with gastric (12) or resected pancreatic cancer (13) in a phase III trial. However, the proper administration protocol for S-1 in the adjuvant setting for completely resected NSCLC is unclear. For patients with gastric cancer, the original schedule of adjuvant S-1 is 4 weeks of administration followed by 2 weeks of withdrawal (14). The conventional administration of S-1 is reportedly difficult to perform, especially in patients older than 65 years with NSCLC. Okumura et al. reported the median relative dose intensity of conventional S-1 of patients over 65 years was lower compared to patients under 65 years $(44.8 \% v s$. $100 \%, \mathrm{P}=0.013$ ), and they concluded conventional schedule of adjuvant chemotherapy with S-1 is not likely to be feasible (15). Several studies were conducted to reduce its toxicity. In the LOGIK 0901 study, the regimen of 2 weeks of administration and 1 week of withdrawal was feasible (16). Sakuma et al. reported that the alternative administration method for S-1 in patients with gastric cancer had reduced toxicity without compromising effectiveness (17).

Based on these findings, four courses of carboplatin plus S-1 followed by S-1 maintenance adjuvant chemotherapy is a promising regimen requiring reduced $\mathrm{S}-1$ administration compared with the conventional method. In the present 


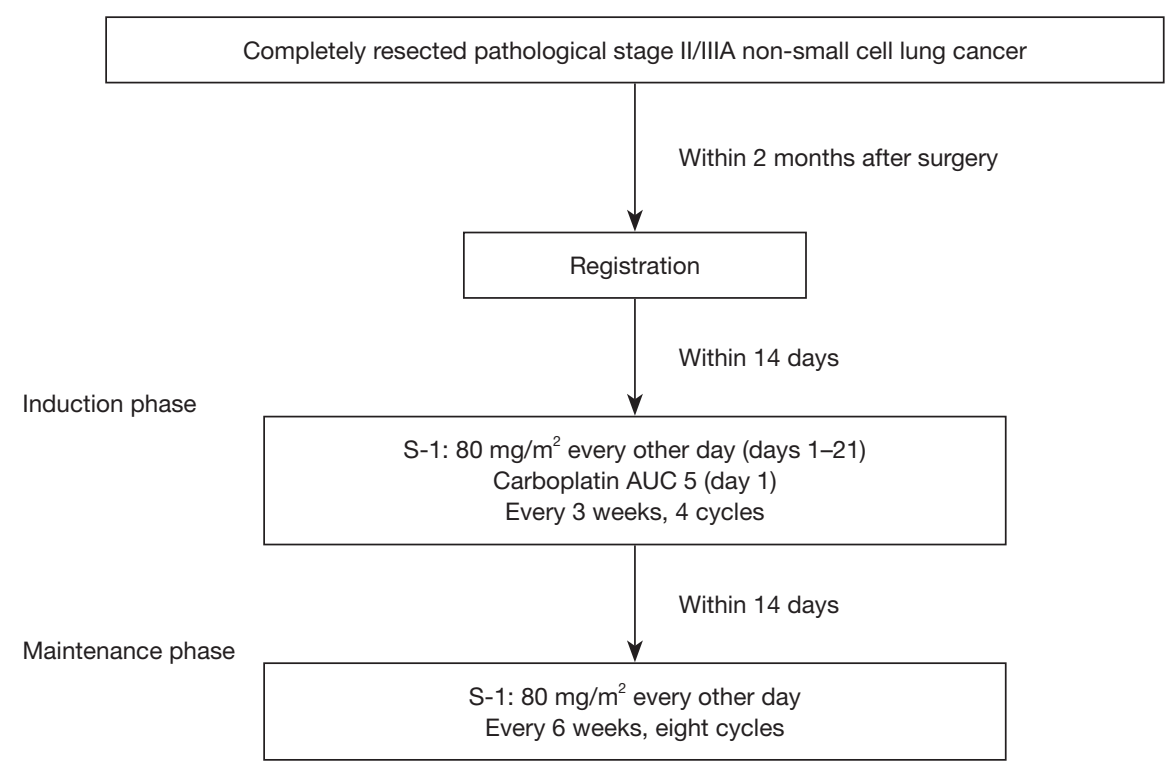

Figure 1 Chemotherapy compliance in the present study. AE, adverse event.

study, we aimed to confirm the tolerability and efficacy of four courses of carboplatin plus S-1 followed by S-1 administration every other day for 1 year.

\section{Methods}

\section{Eligibility criteria}

Eligible patients were 20-75 years old, and their histologically confirmed pathological stage IIA or IIB or IIIA (TNM classification ver. 7) NSCLC was treated via complete resection using lobectomy, bilobectomy, or pneumonectomy with hilar and mediastinal lymph node dissection. Patients were also required to have the ability to start chemotherapy within 2 months after surgery, an Eastern Cooperative Oncology Group Performance Status of $0-1$, and adequate organ function (i.e., total bilirubin $\leq 1.5 \mathrm{mg} / \mathrm{dL}$, aspartate aminotransferase and alanine aminotransferase $\leq 100 \mathrm{IU} / \mathrm{L}$, serum creatinine $\leq 1.5 \mathrm{mg} / \mathrm{dL}$, leukocyte count $\geq 3,000 / \mathrm{mm}^{3}$, neutrophil count $\geq 1,500 / \mathrm{mm}^{3}$, hemoglobin $\geq 8.0 \mathrm{~g} / \mathrm{dL}$, platelets $\geq 100,000 / \mathrm{mm}^{3}$, and $\mathrm{SpO}_{2}$ at room air $\geq 95 \%$ ). The key exclusion criteria were as follows: interstitial pneumonia or pulmonary fibrosis, prior receipt of chemotherapy, severe surgical complications, synchronous or metachronous multiple lung cancer or double cancer in another organ requiring treatment within 5 years of this study, active hepatitis $\mathrm{B}$ or $\mathrm{C}$ virus infection, severe complications such as uncontrollable hypertension or difficult-to-control diabetes mellitus, lactation, and pregnancy or possible pregnancy in women.

\section{Treatment}

This prospective single-arm phase II study was conducted at eight centers belonging to the Japan Northern East Area Thoracic Surgery Study Group (JNETS). Curacy or integrity of any part of the work are appropriately investigated and resolved. This study was undertaken in accordance with Declaration of Helsinki. The study protocol was approved by the institutional review boards of all participating centers, and all patients provided written informed consent. This study was registered with the UMIN Clinical Trial Registry (UMIN000011306). Details are available at the following address: https://upload.umin. ac.jp/cgi-open-bin/ctr/ctr.cgi? function=brows\&action=br ows\&type $=$ summary \& recptno $=$ R000013243\&language $=J$. The study was self-funded. Participants were administered a total dose of carboplatin AUC 5 on day 1 and oral S-1 every other day for 3 weeks. After four courses of carboplatin plus S-1, maintenance oral S-1 every other day for 6 weeks was started within 2 weeks. A total of 48 weeks (eight courses) of oral S-1 were needed to complete maintenance therapy (Figure 1). Patient follow-up was performed by the regional hospitals until at least 2 years after registration. The causes of death were determined by the Data and Safety Monitoring Committee of JNETS after reviewing the patients' medical records if necessary. 
Therapy-related deaths were regarded as deaths from lung cancer. Within 2 months after surgery, eligible patients were assigned to receive adjuvant carboplatin plus S-1 followed by oral maintenance S-1. All adverse events occurring during the present study were recorded by the investigators and graded according to the National Cancer Institute-Common Terminology Criteria for Adverse Events version 4.0 (ver. 4.0). Data were collected, managed, and analyzed by the Department of Chest Surgery, Fukushima Medical University.

\section{Patient follow-up}

Patients were examined via physical examination before and during every treatment course. Computed tomography of the chest and upper abdomen was performed twice per year, and magnetic resonance imaging of the brain was performed once per year according to the study protocol. ${ }^{18} \mathrm{~F}$-Fluorodeoxyglucose positron emission tomography or bone scintigraphy was performed once before patient enrollment in the present study.

\section{Endpoints and statistical analysis}

The primary endpoint was the treatment completion rate (four courses of carboplatin plus S-1 followed by eight courses of maintenance S-1). The secondary endpoints were the 2-year recurrence-free survival (RFS) rate, incidence of adverse events, and probability of worst-grade adverse events.

\section{Statistical analysis}

The survival curve was estimated using the KaplanMeier method. The differences in survival probabilities were compared using the log-rank test. Univariate and multivariate analyses were conducted using Cox's proportional hazard model. A $\mathrm{P}$ value of less than 0.05 was considered statistically significant. We set the threshold of the treatment completion rate at $50 \%$ and expected a treatment completion rate of $70 \%$. With an alpha error of $5 \%$ and a power of $80 \%$, the sample size was calculated as 37 cases. We thus sought to enroll 40 cases including ineligible cases. All statistical analyses were conducted using SPSS statistics 25.0 software for Windows (SPSS Inc., Chicago, IL, USA).

\section{Statement of ethic approval}

This study was undertaken in accordance with Declaration
Table 1 Patient characteristics

\begin{tabular}{lc}
\hline Factor & $\mathrm{N}=40$ \\
\hline Age (years), median [range] & 65 [39-75] \\
Gender (male/female) & $28 / 12$ \\
Histology & Ad 29, Sq 7, others 4 \\
$\begin{array}{l}\text { Tumor burden (mm), median } \\
\text { [range] }\end{array}$ & 33 [15-140] \\
Surgical procedure & Lobectomy 36, bilobectomy 2, \\
Nodal status & pneumonectomy 2 \\
Pleural invasion & N0: 8, N1: 11, N2: 21 \\
Lymph node dissection & ND2a-1: 8, ND2a-2: 32 \\
Pathological stage (UICC7th) & 2A: 12, 2B: 5, 3A: 23 \\
Recurrence & Yes 27, no 13 \\
Prognosis & Dead 7, alive 33 \\
\hline
\end{tabular}

of Helsinki, and it was also approved by the institutional review board of each institution. Written informed consent of surgery and the use of clinical data were obtained from all patients involved in this study.

\section{Results}

\section{Patient characteristics}

Between November 2013 and January 2015, 40 patients from eight institutes in JNETS were registered. Table 1 shows the demographics and characteristics of the 40 patients enrolled in the present study. The median patient age was 65 years (range, 39-75 years), and 13 patients were $\geq 70$ years old. As of July 1, 2018, the median duration of follow-up was 26 months (range, 8-39 months). Male predominance $(70 \%)$ was observed in the present study. Recurrence of lung cancer was detected in 27 patients (67.5\%) during chemotherapy and follow-up, and six and one patient died of lung cancer and aspiration pneumonia, respectively.

\section{Treatment compliance}

Figure 2 shows the treatment compliance rates in the present trial. Overall, 27 patients $(67.5 \%)$ completed the four cycles of carboplatin plus S-1. Meanwhile, nine patients completed both the four planned courses of 
Induction phase (carboplatin AUC 5 on day1 plus oral S-1 every other day for 3 weeks; total four courses)
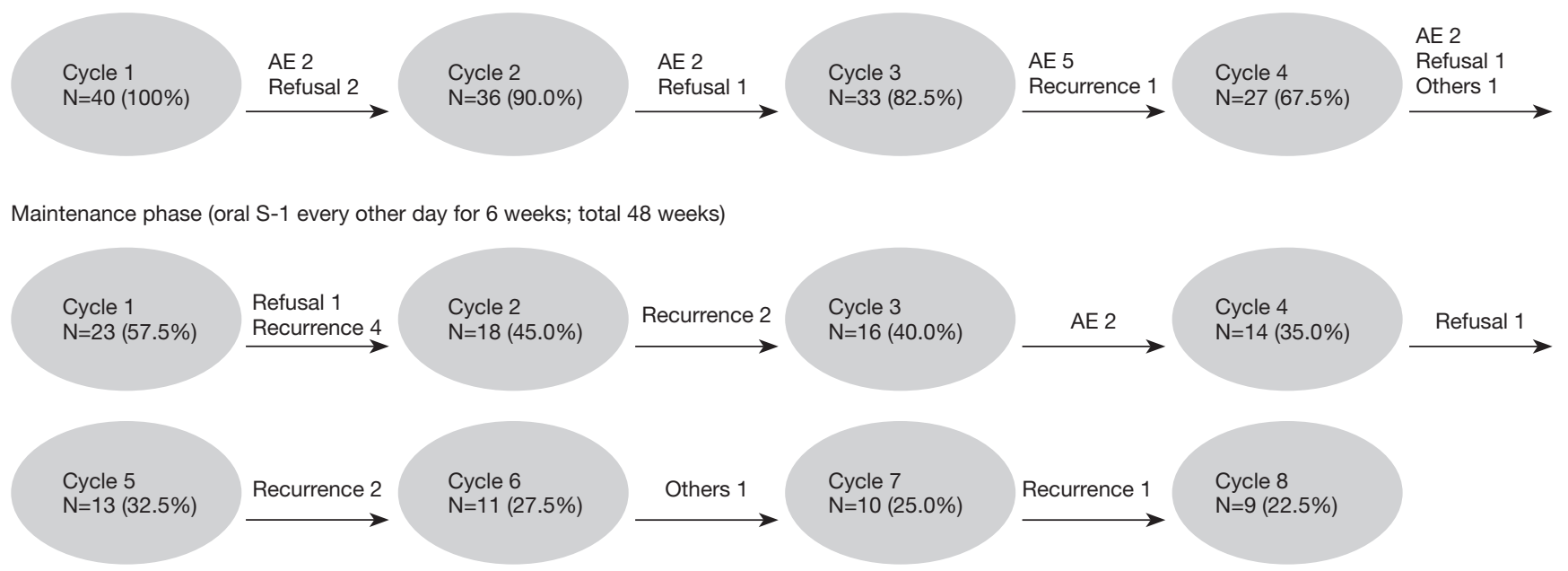

Figure 2 CONSORT diagram of patient flow in the present study. AUC, area under the curve.

carboplatin plus S-1 and eight courses of maintenance S-1. Ten patients were censored because of disease recurrence; therefore, the treatment completion rate was calculated as $30 \%$ [ $90 \%$ confidence interval (CI): 40.3-63.0\%). The reasons for patient withdrawal or treatment discontinuation during the induction phase were adverse events $(n=9$ : neutropenia, 6; thrombocytopenia, 1; pancytopenia, 2; peripheral neuropathy, 1; and anorexia, 1), patient refusal $(n=3)$, and relapse of cancer $(n=1)$. The lower bound of the $90 \%$ CI of $40.3 \%$ was lower than we expected before starting the present study. The reasons for discontinuing S-1 maintenance therapy were adverse events $(n=4$ : one case each of hyperbilirubinemia, hematological examination disorder, fatigue, and eruption), patient refusal $(\mathrm{n}=3)$, relapse of cancer $(n=9)$, and other reasons $(n=2$ : early cessation of maintenance chemotherapy because of misunderstanding by the responsible doctor regarding the chemotherapy course, 1 ; and cerebral hemorrhage not related to chemotherapy, 1).

\section{Survival analysis}

The Kaplan-Meier curve for 2-year RFS is shown in Figure 3. The 2-year RFS rate of all patients registered in this study was $31.1 \%$ (data not shown). Patients who completed adjuvant carboplatin plus S-1 followed by maintenance S-1 had a significantly better RFS rate than patients who discontinued treatment $(66.7 \%$ vs. $30.5 \%$, $\mathrm{P}=0.033)$. Univariate analysis for 2 -year RFS identified completion of maintenance S-1 chemotherapy as a prognostic factor (hazard ratio $=0.283,95 \%$ CI: $0.080-$ $0.995, \mathrm{P}=0.049$, Table 2), although it did not remain a significant factor in multivariate analysis. The 2-year OS rate did not significantly differ according to the completion of the scheduled maintenance regimen $(100 \%$ vs. $84.6 \%$, $\mathrm{P}=0.227$, data not shown), but patients who completed maintenance chemotherapy did not die during the followup period.

\section{Adverse events}

All adverse events that developed during the present study are listed in Table 3. There were no treatment-related deaths. Hematological disorders, especially cytopenia, were frequently observed during the administration of carboplatin plus S-1. Febrile neutropenia did not occur during the S-1 maintenance phase. Other disorders included mild liver or renal dysfunction, which did not require intensive treatment. Anorexia $(52.5 \%)$ and nausea $(42.5 \%)$ were frequent digestive adverse events, whereas vomiting was rarely observed in the present study. Digestive adverse events were not observed in the S-1 maintenance phase. The atypical G3 adverse events included two cases of vagal reflex in the carboplatin plus S-1 phase and two cases of rash in the S-1 maintenance phase. The only grade 4 adverse events were seven cases of neutropenia that were managed well via the subcutaneous injection of granulocyte 

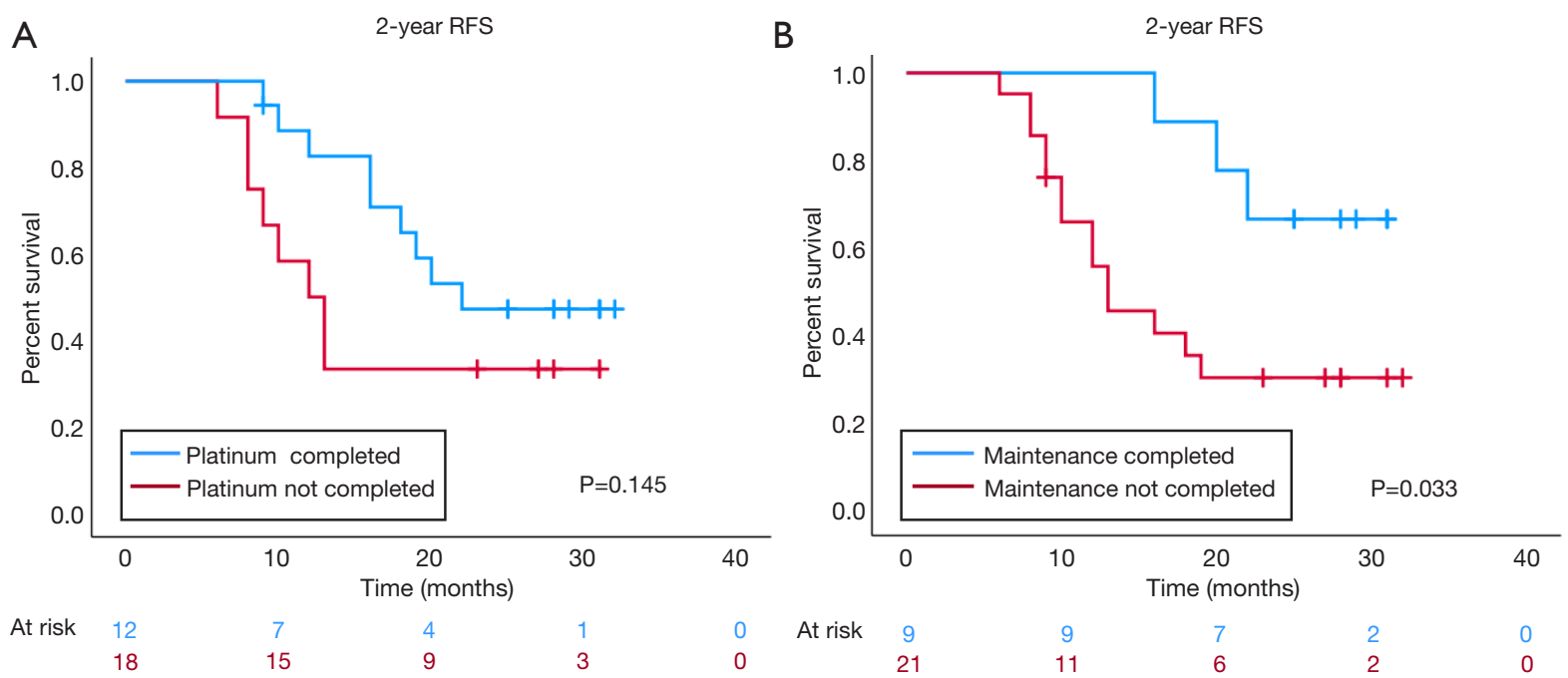

Figure 3 Two-year recurrence-free survival (RFS) according to the completion of four courses of carboplatin plus S-1 (A) and following eight courses of maintenance of S-1 (B).

Table 2 Univariate and multivariate analyses of 2-year recurrence-free survival using a Cox proportional hazard model

\begin{tabular}{|c|c|c|c|c|c|c|c|}
\hline \multirow{2}{*}{ Factor } & \multirow{2}{*}{ Favorable } & \multicolumn{3}{|c|}{ Univariate } & \multicolumn{3}{|c|}{ Multivariate } \\
\hline & & $\mathrm{HR}$ & $95 \% \mathrm{Cl}$ & $P$ value & $\mathrm{HR}$ & $95 \% \mathrm{Cl}$ & $P$ value \\
\hline Age & $<65$ years & 0.979 & $0.377-2.540$ & 0.965 & 1.119 & $0.321-3.898$ & 0.860 \\
\hline Gender & Female & 0.780 & $0.224-2.723$ & 0.697 & 0.382 & $0.082-1.783$ & 0.221 \\
\hline Histology & Adeno & 1.675 & $0.480-5.843$ & 0.418 & 1.735 & $0.284-10.605$ & 0.551 \\
\hline PI invasion & $<3$ & 0.183 & $0.038-0.890$ & 0.035 & 0.498 & $0.062-3.870$ & 0.498 \\
\hline Multistation LN metastasis & Negative & 0.338 & $0.110-1.042$ & 0.059 & 1.376 & $0.176-10.753$ & 0.761 \\
\hline p-stage & II & 0.256 & $0.082-0.793$ & 0.018 & 0.174 & $0.022-1.368$ & 0.096 \\
\hline Platinum chemotherapy & Completion & 0.503 & $0.193-1.312$ & 0.160 & 0.541 & $0.121-2.414$ & 0.421 \\
\hline
\end{tabular}

HR, hazard ratio; Cl, confidence interval; PL, pleural; LN, lymph node.

colony stimulate factor. In the maintenance phase, two grade 3 adverse events were observed (one general fatigue, one dyspnea).

\section{Discussion}

The present study was conducted to confirm the feasibility and efficacy of carboplatin plus S-1 followed by maintenance S-1 regarding 2-year RFS and the proportion of adverse events in patients with completely resected stage II/IIIA NSCLC. The LETS study reported that the carboplatin plus S-1 arm had lower toxicity and higher dose intensity than the carboplatin plus paclitaxel arm (5); however, the treatment completion rate in the present study was $30.0 \%$, which was lower than we expected before starting the present study based on prior findings using similar carboplatin plus S-1 regimens. In our study, severe adverse events necessitating intensive care were not observed. The 2-year RFS rate, which was a secondary endpoint of this study, was $66.7 \%$ in the maintenance chemotherapy completed group, which was non-inferior to that reported previously for carboplatin plus S-1. 
Table 3 List of adverse events and proportion of patients with the worst grade $(\mathrm{N}=40)$

\begin{tabular}{|c|c|c|c|}
\hline Adverse event & Any grade (\%) & Worst grade (\%) & Worse than G3 (\%) \\
\hline Leukocytopenia & $20(50.0)$ & $3(2.5)$ & $1(2.5)$ \\
\hline Neutropenia & $23(57.5)$ & $4(5.0)$ & $3(7.5)$ \\
\hline Thrombocytopenia & $24(60.0)$ & $3(2.5)$ & $1(2.5)$ \\
\hline AST elevation & $6(15.0)$ & $1(15.0)$ & $0(0.0)$ \\
\hline ALT elevation & $9(22.5)$ & $1(22.5)$ & $0(0.0)$ \\
\hline Hyperbilirubinemia & $11(27.5)$ & $2(5.0)$ & $0(0.0)$ \\
\hline Creatinine increased & $12(30.0)$ & $2(5.0)$ & $0(0.0)$ \\
\hline Hypokalemia & 9 (22.5) & $3(2.5)$ & $1(2.5)$ \\
\hline \multicolumn{4}{|l|}{ Non-hematological } \\
\hline Fever & $4(10.0)$ & $1(10.0)$ & $0(0.0)$ \\
\hline Fatigue & $21(52.5)$ & $3(7.5)$ & $3(7.5)$ \\
\hline Anorexia & $21(52.5)$ & $3(2.5)$ & $1(2.5)$ \\
\hline Weight loss & $11(27.5)$ & $2(2.5)$ & $0(0.0)$ \\
\hline Constipation & $17(42.5)$ & $2(7.5)$ & $0(0.0)$ \\
\hline Diarrhea & $9(22.5)$ & $2(5.0)$ & $0(0.0)$ \\
\hline Dysgeusia & $8(20.0)$ & $1(20.0)$ & $0(0.0)$ \\
\hline Peripheral sensory neuropathy & 7 (17.5) & $3(2.5)$ & $1(2.5)$ \\
\hline Muscle pain & $1(2.5)$ & $1(2.5)$ & $0(0.0)$ \\
\hline Arthralgia & $0(0.0)$ & $0(0.0)$ & $0(0.0)$ \\
\hline Upper respiratory infection & $2(5.0)$ & $1(5.0)$ & $0(0.0)$ \\
\hline Pulmonary infection & $1(2.5)$ & $3(2.5)$ & $1(2.5)$ \\
\hline Febrile neutropenia & $1(2.5)$ & $3(2.5)$ & $1(2.5)$ \\
\hline Dyspnea & $1(2.5)$ & $3(2.5)$ & $1(2.5)$ \\
\hline Eruption & $2(5.0)$ & $3(2.5)$ & $1(2.5)$ \\
\hline Vagal reflex & $1(2.5)$ & $3(2.5)$ & $1(2.5)$ \\
\hline Orthostatic hypotension & $1(2.5)$ & $3(2.5)$ & $1(2.5)$ \\
\hline Alopecia & $0(0.0)$ & $0(0.0)$ & $0(0.0)$ \\
\hline
\end{tabular}

AST, aspartate aminotransferase; ALT, alanine aminotransferase. 
This study did not achieve the expected treatment completion rate. The causes of the low treatment completion rate differed between the two treatment phases. In the carboplatin plus S-1 phase, treatment discontinuation was mainly caused by adverse events, especially in patients older than 65 years. Although grade 3-4 hematological or non-hematological adverse events were infrequent in the present study, prolonged fatigue and/or anorexia were too difficult for patients who recently underwent radical resection for NSCLC to endure. This phenomenon was probably attributable to CDHP clearance prolongation in elderly patients as previously described. Fujita $e t$ al. reported the median AUC of CDHP was significantly higher in patients 75 years or older than in those younger than 75 years although AUC of active 5-FU did not significantly differ between patients 75 years or older and younger than 75 years. This effect was caused by reduced renal function in elderly, because CDHP is excreted in the urine by glomerular filtration (18). We speculated that the age at the time of surgery is one of the most important issues affecting the success of clinical studies, especially studies of cytotoxic chemotherapy, from the viewpoints of both physiological function and weakness because of adverse events. In the present study, 13 patients (32.5\%) were older than 70 years at the time of registration, and only two of these patients completed the planned treatment. Conversely, the main reason for discontinuing oral S-1 was recurrence of lung cancer. Twenty-seven patients continued maintenance chemotherapy, nine of whom experienced relapse in the maintenance phase, which was one reason for the low treatment completion rate of the present study. Tumor relapse in the maintenance phase was more common than we expected when we started the present study. Although the treatment completion rate was low, the 2-year RFS rate of the patients who completed the protocol treatment was not inferior to previously reported results for adjuvant chemotherapy using carboplatin plus S-1.

From the viewpoint of maintenance chemotherapy, four courses of cisplatin plus pemetrexed followed by maintenance tri-weekly pemetrexed statistically prolonged OS compared with the results of a protocol lacking maintenance therapy in the PARAMOUNT study (7). The JMEN study observed prolonged OS for maintenance pemetrexed therapy after four courses of platinum-based chemotherapy (8). In the SATURN study, four courses of platinum doublet chemotherapy followed by maintenance erlotinib also statistically prolonged OS compared with the survival rate without maintenance therapy (9). Recently, a randomized phase II study of adjuvant chemotherapy for patients with completely resected pathological stage II/ III NSCLC was conducted by the West Japan Oncology Group (WJOG4107). Their study protocol was 2 weeks of oral S-1 followed by 1 week of withdrawal for 1 year $v s$. four courses of cisplatin plus S-1. The 2-year RFS and treatment completion rates for oral S-1 were $65.6 \%$ and $52.6 \%$, respectively, vs. $58.1 \%$ and $74.7 \%$, respectively, for cisplatin plus S-1 (19). Niho et al. reported a phase II study of two courses of cisplatin $\left(80 \mathrm{mg} / \mathrm{m}^{2}\right.$, day 1$)$ and docetaxel $\left(60 \mathrm{mg} / \mathrm{m}^{2}\right.$, day 1) followed by single-agent therapy with S-1 [40 mg/m², twice daily for 14 consecutive days, for $>6$ months (maximum 1 year)] as adjuvant chemotherapy for completely resected stage II-III NSCLC, and the treatment completion and 2-year RFS rates were 51.2\% and $75.9 \%$, respectively (20). Okumura et al. reported a protocol consisting of four courses of carboplatin plus S-1 followed by maintenance S-1 for 10 months as adjuvant chemotherapy, and the treatment completion and 2-year RFS rates were $63.2 \%$ (90\% CI: $54.4-71.2 \%$ ) and 59.8\%, respectively (21). Okamoto et al. treated patients with completely resected stage II/III NSCLC with long-term S-1 monotherapy or S-1 plus cisplatin followed by S-1 for 1 year. The 2-year RFS and treatment completion rates in the S-1 monotherapy arm were 52\% (95\% CI: 0.40-0.63) and $45.7 \%$ (95\% CI: 41.9-66.3\%), respectively, vs. $61 \%$ (95\% CI: $0.48-0.70)$ and $43.5 \%$ (95\% CI: $44.0-68.4 \%$ ), respectively, for the combination regimen (22). The 2 -year RFS rate in our study was comparable to previous findings despite our inferior treatment completion rate. Previous studies of adjuvant chemotherapy for completely resected NSCLC using S-1 suggested that the regimen was relatively intolerable as an alternative conventional cisplatin regimen. One of the most important issues for this regimen is its tolerability as adjuvant chemotherapy for NSCLC. Regarding conventional cisplatin-based doublet adjuvant chemotherapy, treatment completion rates of $40-50 \%$ have been reported $(1-3,23)$, and these results did not differ from those for S-1 as adjuvant chemotherapy $(20,22)$. Lipkin et al. (24) and Clarkson et al. (25) reported a large difference in the cell cycle between normal host cells and cancer cells. That is, the cell cycle of host normal cells is approximately 12-24 $\mathrm{h}$ in duration, and S-phase, during which 5-FU exerts its effects, requires approximately $12 \mathrm{~h}$ for completion. Therefore, when considering the clinically optimal administration method, avoidance of exposure of 5-FU for $24 \mathrm{~h}$ will protect considerable numbers of normal cells from toxicity such as damage of intestinal mucosa. In addition, 
because the cell cycle of cancer cells is 4-5 days and S-phase is longer than $24 \mathrm{~h}$, even if $5-\mathrm{FU}$ is repeatedly administered every other day, it will be possible to attenuate cytotoxic effects of chemotherapy. Shirasaka et al. recommended the administration of S-1 every other day for this reason (26). In patients with gastric cancer, alternate-day S- 1 administration is a standard adjuvant chemotherapy regimen with reduced toxicity $(17,27)$. Hata et al. reported that an S-1 regimen consisting of 2 weeks of treatment followed by 1 week of withdrawal was not superior to the conventional regimen of 4 weeks of administration followed by 2 weeks of withdrawal regarding treatment compliance (28). Taking these findings into account, alternate-day administration of S-1 with subsequent maintenance chemotherapy could be a reasonable and promising regimen. However, in clinical practice, adjuvant chemotherapy using S-1 for 1 year was less tolerable than anticipated. Clinical biomarkers that can predict feasibility or adverse events related to S-1 are not available in current practice. These previous trials and our present findings suggest that adjuvant chemotherapy regimens containing $\mathrm{S}-1$ are promising, but problems regarding treatment compliance persist, especially among elderly patients.

This study had several limitations. First, the number of patients was small, and the follow-up period was short. A larger difference in OS might be observed after long-term follow-up. Second, this study was a single-arm phase II study, and thus, its results may not be robust.

In conclusion, four courses of carboplatin plus S-1 followed by 1 year of maintenance S-1 every other day as adjuvant chemotherapy for patients with pathological stage II/IIIA NSCLC was not feasible using our study design; however, patients who can fully complete the regimen might experience clinical benefit. Clinical biomarkers might be needed to select such patients.

\section{Acknowledgments}

We thank Joe Barber Jr, PhD, from Edanz Group (www. edanzediting.com/ac) for editing a draft of this manuscript. Funding: None.

\section{Footnote}

Data Sharing Statement: Available at http://dx.doi. org/10.21037/jtd-20-715

Conflicts of Interest: All authors have completed the ICMJE uniform disclosure form (available at http://dx.doi. org/10.21037/jtd-20-715). The authors have no conflicts of interest to declare.

Ethical Statement: The authors are accountable for all aspects of the work in ensuring that questions related to the accuracy or integrity of any part of the work are appropriately investigated and resolved. This study was undertaken in accordance with Declaration of Helsinki (as revised in 2013), and it was also approved by the institutional review board of each institution (No. 1775). Written informed consent of surgery and the use of clinical data were obtained from all patients involved in this study.

Open Access Statement: This is an Open Access article distributed in accordance with the Creative Commons Attribution-NonCommercial-NoDerivs 4.0 International License (CC BY-NC-ND 4.0), which permits the noncommercial replication and distribution of the article with the strict proviso that no changes or edits are made and the original work is properly cited (including links to both the formal publication through the relevant DOI and the license). See: https://creativecommons.org/licenses/by-nc-nd/4.0/.

\section{References}

1. Pignon JP, Tribodet H, Scagliotti GV, et al. Lung adjuvant cisplatin evaluation: a pooled analysis by the LACE Collaborative Group. J Clin Oncol 2008;26:3552-9.

2. Douillard JY, Rosell R, De Lena M, et al. Adjuvant vinorelbine plus cisplatin versus observation in patients with completely resected stage IB-IIIA non-small-cell lung cancer (Adjuvant Navelbine International Trialist Association [ANITA]): a randomised controlled trial. Lancet Oncol 2006;7:719-27.

3. Winton T, Livingston R, Johnson D, et al. Vinorelbine plus cisplatin vs. observation in resected non-small-cell lung cancer. N Engl J Med 2005;352:2589-97.

4. Strauss GM, Herndon JE 2nd, Maddaus MA, et al. Adjuvant paclitaxel plus carboplatin compared with observation in stage IB non-small-cell lung cancer: CALGB 9633 with the Cancer and Leukemia Group B, Radiation Therapy Oncology Group, and North Central Cancer Treatment Group Study Groups. J Clin Oncol 2008;26:5043-51.

5. Yoshioka H, Okamoto I, Morita S, et al. Efficacy and safety analysis according to histology for S-1 in combination with carboplatin as first-line chemotherapy in patients with 
advanced non-small-cell lung cancer: updated results of the West Japan Oncology Group LETS study. Ann Oncol 2013;24:1326-31.

6. Chang WJ, Sun JM, Lee JY, et al. A retrospective comparison of adjuvant chemotherapeutic regimens for non-small cell lung cancer (NSCLC): paclitaxel plus carboplatin versus vinorelbine plus cisplatin. Lung Cancer 2014;84:51-5.

7. Paz-Ares L, de Marinis F, Dediu M, et al. Maintenance therapy with pemetrexed plus best supportive care versus placebo plus best supportive care after induction therapy with pemetrexed plus cisplatin for advanced non-squamous non-small-cell lung cancer (PARAMOUNT): a doubleblind, phase 3, randomised controlled trial. Lancet Oncol 2012;13:247-55.

8. Ciuleanu T, Brodowicz T, Zielinski C, et al. Maintenance pemetrexed plus best supportive care versus placebo plus best supportive care for non-small-cell lung cancer: a randomised, double-blind, phase 3 study. Lancet 2009;374:1432-40.

9. Cappuzzo F, Ciuleanu T, Stelmakh L, et al. Erlotinib as maintenance treatment in advanced non-small-cell lung cancer: a multicentre, randomised, placebo-controlled phase 3 study. Lancet Oncol 2010;11:521-9.

10. Shirasaka T, Nakano K, Takechi T, et al. Antitumor activity of $1 \mathrm{M}$ tegafur-0.4 M 5-chloro-2,4-dihydroxypyridine-1 M potassium oxonate (S-1) against human colon carcinoma orthotopically implanted into nude rats. Cancer Res 1996;56:2602-6.

11. Tanaka F, Wada H, Fukushima M. UFT and S-1 for treatment of primary lung cancer. Gen Thorac Cardiovasc Surg 2010;58:3-13.

12. Sakuramoto S, Sasako M, Yamaguchi T, et al. Adjuvant chemotherapy for gastric cancer with S-1, an oral fluoropyrimidine. N Engl J Med 2007;357:1810-20.

13. Uesaka K, Boku N, Fukutomi A, et al. Adjuvant chemotherapy of S-1 versus gemcitabine for resected pancreatic cancer: a phase 3, open-label, randomised, noninferiority trial (JASPAC 01). Lancet 2016;388:248-57.

14. Kinoshita T, Nashimoto A, Yamamura Y, et al. Feasibility study of adjuvant chemotherapy with S-1 (TS-1; tegafur, gimeracil, oteracil potassium) for gastric cancer. Gastric Cancer 2004;7:104-9.

15. Okumura S, Sasaki T, Satoh K, et al. Feasibility of adjuvant chemotherapy with S-1 consisting of a 4-week administration and a two-week rest period in patients with completely resected non-small cell lung cancer. Mol Clin Oncol 2013;1:124-30.
16. Maruyama R, Ebi N, Kishimoto J, et al. A feasibility trial of postoperative adjuvant chemotherapy with S-1, an oral fluoropyrimidine, for elderly patients with non-small cell lung cancer: a report of the Lung Oncology Group in Kyushu (LOGIK) protocol 0901. Int J Clin Oncol 2014;19:57-62.

17. Sakuma K, Hosoya Y, Arai W, et al. Alternate-day treatment with S-1 in patients with gastric cancer: a retrospective study of strategies for reducing toxicity. Int J Clin Oncol 2010;15:166-71.

18. Fujita K, Nakayama H, Ichikawa W, et al. Pharmacokinetics of 5 -fluorouracil in elderly Japanese patients with cancer treated with S-1 (a combination of tegafur and dihydropyrimidine dehydrogenase inhibitor 5-chloro-2,4-dihydroxypyridine). Drug Metab Dispos 2009;37:1375-7.

19. Iwamoto Y, Mitsudomi T, Sakai K, et al. Randomized Phase II Study of Adjuvant Chemotherapy with Longterm S-1 versus Cisplatin $+\mathrm{S}-1$ in Completely Resected Stage II-IIIA Non-Small Cell Lung Cancer. Clin Cancer Res 2015;21:5245-52.

20. Niho S, Ikeda N, Michimae H, et al. Overall Survival Results of the Feasibility Study of Adjuvant Chemotherapy With Docetaxel Plus Cisplatin Followed by Long-term Single-agent Administration of S-1 in Patients With Completely Resected Non-Small Cell Lung Cancer: Thoracic Oncology Research Group (TORG) 0809. Am J Clin Oncol 2018;41:1113-7.

21. Okumura N, Sonobe M, Okabe K, et al. Feasibility of adjuvant chemotherapy with S-1 plus carboplatin followed by single-agent maintenance therapy with $S-1$ for completely resected non-small-cell lung cancer: results of the Setouchi Lung Cancer Group Study 1001. Int J Clin Oncol 2017;22:274-82.

22. Okamoto T, Yano T, Shimokawa M, et al. A phase II randomized trial of adjuvant chemotherapy with $\mathrm{S}-1$ versus S-1 plus cisplatin for completely resected pathological stage II/IIIA non-small cell lung cancer. Lung Cancer 2018;124:255-9.

23. Tanvetyanon T, Lee JH, Fulp WJ, et al. Use of Adjuvant Cisplatin-Based Versus Carboplatin-Based Chemotherapy in Non-Small-Cell Lung Cancer: Findings From the Florida Initiative for Quality Cancer Care. J Oncol Pract 2015;11:332-7.

24. Lipkin M, Sherlock P, Bell B. Cell proliferation kinetics in the gastrointestinal tract of man. II. cell renewal in stomach, ileum, colon, and rectum. Gastroenterology 1963;45:721-9. 
25. Clarkson B, Ota K, Ohkita T, et al. Kinetics of proliferation of cancer cells in neoplastic effusions in man. Cancer 1965;18:1189-213.

26. Shirasaka T, Yamamitsu S, Tsuji A, et al. Conceptual changes in cancer chemotherapy: from an oral fluoropyrimidine prodrug, UFT, to a novel oral fluoropyrimidine prodrug, S-1, and low-dose FP therapy in Japan. Invest New Drugs 2000;18:315-29.

27. Tatebe S, Tsujitani S, Nakamura S, et al. Feasibility study

Cite this article as: Hasegawa T, Suzuki H, Abe J, Sakurada A, Endo C, Sato N, Hasumi T, Deguchi H, Oura H, Takahashi S, Saito H, Uramoto H, Sagawa M, Okada Y. A phase 2 study of adjuvant carboplatin plus S-1 followed by maintenance S-1 therapy for patients with completely resected stage II/IIIA non-small cell lung cancer-Japanese Northern East Area Thoracic Surgery Study Group JNETS1302 study. J Thorac Dis 2020;12(7):3591-3601. doi: 10.21037/jtd-20-715 of alternate-day S-1 as adjuvant chemotherapy for gastric cancer: a randomized controlled trial. Gastric Cancer 2014;17:508-13.

28. Hata Y, Kiribayashi T, Kishi K, et al. Adherence and feasibility of 2 treatment schedules of S-1 as adjuvant chemotherapy for patients with completely resected advanced lung cancer: a multicenter randomized controlled trial. BMC Cancer 2017;17:581. 